\title{
A Note on Elastic Surface Waves
}

\author{
J. K. KNOWLES \\ Division of Engineering and Applied Science \\ California Institute of Technology, Pasadena
}

The structure of harmonically time-dependent free surface waves on a homogeneous, isotropic elastic half-space can be described by proceeding from the following assumptions: (1) the plane boundary is free of surface traction; (2) the Lamé potentials, and consequently all physical quantities, decay exponentially with distance away from the boundary. In the absence of further a priori assumptions, the resulting surface waves need be neither plane nor axially symmetric, and thus the derivation sketched here constitutes a generalization of the ones usually given in the textbook literature [e.g., Love, 1944; Ewing et al., 1957].

With reference to Cartesian coordinates $x_{1}$, $x_{2}, x_{3}$, the half-space under consideration occupies the region $x_{3} \geq 0$. The displacement vector $\mathbf{u}$ of a typical point has Cartesian components $u$, and the associated components of stress are denoted by $\tau_{j k}$. The summation convention is used, Latin and Greek subscripts have the respective ranges $1,2,3$ and 1,2 , and a subscript preceded by a comma indicates differentiation with respect to the corresponding coordinate.

In the absence of body forces, the displacement field may be represented in the form

$$
\mathbf{u}=\nabla \Phi+\nabla \times \mathbf{\Psi}
$$

where $\Phi$ and $\Psi$ are the scalar and vector Lame potentials, respectively. They must satisfy the wave equations

$$
c_{1}^{2} \nabla^{2} \Phi=\frac{\partial^{2} \Phi}{\partial t^{2}} \quad c_{2}^{2} \nabla^{2} \Psi=\frac{\partial^{2} \Psi}{\partial t^{2}}
$$

where $c_{1}$ and $c_{2}<c_{1}$ are the respective speeds of propagation of dilatation and shear waves. The stresses associated with (1) may be calculated

$1 \Psi^{*}$ may be further restricted by requiring that $\nabla \cdot \Psi=0$, but we do not impose this requirement here. For a discussion of the representation theorem underlying (1) and (2), see the paper of Sternberg 1960]. from the relations

$$
\begin{aligned}
\tau_{i k}=\rho\left(c_{1}{ }^{2}-2 c_{2}{ }^{2}\right) & u_{m, m} \delta_{i k} \\
& +\rho c_{2}{ }^{2}\left(u_{2, k}+u_{k, 2}\right)
\end{aligned}
$$

where the customary Lamé moduli have been expressed in terms of $c_{1}, c_{2}$, and the mass density $\rho$. The free surface condition at $x_{3}=0$ requires that

$$
\tau_{3 k}=0 \text { at } x_{3}=0
$$

We consider all possible motions of the halfspace for which $\Phi$ and the components $\Psi_{k}$ of $\Psi$ have the form

$$
\begin{aligned}
\Phi & =\exp \left(i \omega t-a x_{3}\right) \varphi\left(x_{1}, x_{2}\right) \\
\Psi_{k} & =\exp \left(i \omega t-b x_{3}\right) \psi_{k}\left(x_{1}, x_{2}\right)
\end{aligned}
$$

where $a$ and $b$ are complex constants with positive real parts and $\omega$ is the given frequency of the waves. We expressly avoid further assumptions concerning the form of $\varphi$ and $\psi_{k}$, so that we are not restricted, for example, to plane waves.

If we now assume that $\Phi$ and $\Psi$ in (5) satisfy (2) and fulfill the requirement of vanishing shear stress $\tau_{s a}=0$ at $x_{2}=0$, it follows from a calculation too lengthy to be included here that $\varphi$ and $\psi_{3}$ can be expressed in terms of $\psi_{1}, \psi_{2}$ and an as yet undetermined constant $c$ by the formulas

$$
\begin{aligned}
\varphi & =-\frac{c}{\omega}\left(1-\frac{c^{2}}{c_{1}^{2}}\right)^{-1 / 2}\left(1-\frac{1}{2} \frac{c^{2}}{c_{2}^{2}}\right) \epsilon_{\alpha \beta} \psi_{\alpha, \beta} \\
\psi_{3} & =(c / \omega)\left(1-c^{2} / c_{2}^{2}\right)^{1 / 2} \psi_{\beta, \beta}
\end{aligned}
$$

where

$a=\frac{\omega}{c}\left(1-\frac{c^{2}}{c_{1}^{2}}\right)^{1 / 2} \quad b=\frac{\omega}{c}\left(1-\frac{c^{2}}{c_{2}^{2}}\right)^{1 / 2}$

In (6) and (7), $\epsilon_{\alpha \beta}$ is the two-dimensional alternator defined by $\epsilon_{11}=\epsilon_{22}=0, \epsilon_{12}=-\epsilon_{21}=$

2 If the factor $\exp \left(-b x_{s}\right)$ in (5) is replaced by $\exp \left(-b_{k} x_{3}\right)$, where the $b_{k}$ 's are not required in advance to be equal, it can in fact be shown that $b_{1}=b_{2}=b_{3}$ is necessary. 
1. A further consequence of the omitted calculation is that $\psi_{1}$ and $\psi_{2}$ must satisfy the reduced wave equation

$$
\Delta \psi_{\alpha}+\left(\omega^{2} / c^{2}\right) \psi_{\alpha}=0
$$

where $\Delta \equiv \partial^{2} / \partial x_{\beta} \quad \partial x_{\beta}$ is the two-dimensional Laplacian.

The as yet unused condition of vanishing normal stress $\tau_{3 s}=0$ at $x_{3}=0$ now serves only to show that $c$ in (6), (7), and (8) must satisfy the usual equation for the speed of Rayleigh waves:

$$
\left(2-\frac{c^{2}}{c_{2}^{2}}\right)^{2}-4\left(1-\frac{c^{2}}{c_{1}^{2}}\right)^{1 / 2}\left(1-\frac{c^{2}}{c_{2}^{2}}\right)^{1 / 2}=0
$$

A recent detailed analysis of (9) may be found in the note by Hayes and Rivlin [1962], where it is shown that there is precisely one value of $c^{2}$ satisfying (9) for which $a$ and $b$ in (7) have positive real parts.

Equations 6 to 9 are necessary conditions for the satisfaction of (2) and (4) by potentials of the form (5). The fact that they are also sufficient is easily verified.

The displacements which follow from (5), (6), and (7) turn out to involve $\psi_{1}$ and $\psi_{2}$ only in the combination $\chi=\epsilon_{\alpha \beta} \psi_{\alpha, \beta}$. They are given by

$$
\begin{aligned}
& u_{\alpha}=(c / \omega) f\left(x_{3}\right) e^{i \omega t} \chi_{, \alpha}\left(x_{1}, x_{2}\right) \\
& u_{3}=g\left(x_{3}\right) e^{i \omega t} \chi\left(x_{1}, x_{2}\right)
\end{aligned}
$$

where

$$
\begin{gathered}
f\left(x_{3}\right)=-\left(1-\frac{c^{2}}{c_{1}^{2}}\right)^{-1 / 2}\left(1-\frac{c^{2}}{2 c_{2}^{2}}\right) e^{-a x_{3}} \\
+\left(1-\frac{c^{2}}{c_{2}^{2}}\right)^{1 / 2} e^{-b x_{3}} \\
g\left(x_{3}\right)=\left(1-c^{2} / 2 c_{2}^{2}\right) e^{-a x_{3}}-e^{-b x_{3}}
\end{gathered}
$$

Thus the displacements, and consequently all physical quantities, are expressed in terms of a single 'potential' $\chi$ which satisfies the two-dimensional reduced wave equation

$$
\Delta x+\left(\omega^{2} / c^{2}\right) x=0
$$

where $c$ represents the speed of Rayleigh waves.

Let $\chi$ be a complex-valued solution of (12), and let $R\left(x_{1}, x_{2}\right)=\left|\chi\left(x_{1}, x_{2}\right)\right|, \Theta\left(x_{1}, x_{2}\right)=\arg$ $\chi\left(x_{1}, x_{2}\right)$, so that

$$
x=R e^{i \theta}
$$

After inserting (13) into (10) and taking real parts, we obtain the corresponding physical displacements $u_{j}$. It is easily shown by eliminating the time $t$ from the resulting formulas that

$$
\begin{aligned}
& \theta_{, 2} u_{1}-\theta_{, 1} u_{2} \\
& +\frac{c f}{\omega g}\left(\frac{R_{, 2} \theta_{, 1}-R_{, 1} \theta_{, 2}}{R}\right) u_{3}=0
\end{aligned}
$$

This is the equation, in a local Cartesian system of coordinates $u_{1}, u_{2}, u_{3}$, of a plane containing the path of a particle located at $x_{1}, x_{2}, x_{3}$ in the undeformed state. It can further be shown that the particle path in this plane is an ellipse.

The orientation of the plane (14) will change from point to point in the half-space, because $R$ and $\Theta$ depend on $x_{1}, x_{2}$, and $f$ and $g$ depend on $x_{s}$. The plane will be normal to the boundary $x_{3}=0$ if and only if the coefficient of $u_{3}$ in (14) vanishes. For the familiar special case of plane waves, we can take

$$
\chi=e^{ \pm i \omega \pi_{1} / c}
$$

without loss of generality, so that $R \equiv 1, \theta=$ $\pm \omega x_{1} / c$. Since $R$ is constant, the coefficient of $u_{3}$ in (14) vanishes, so that the plane of motion is indeed normal to the boundary plane.

In the case of axially symmetric surface waves, the appropriate solutions of (12) are expressible in terms of Hankel functions of order zero; calculation shows that the plane of motion is again normal to the plane $x_{3}=0$.

An example of a solution of (12) for which the corresponding plane (14) is not perpendicular to the boundary is provided by

$$
\chi=H_{n}^{(1,2)}(\omega r / c) e^{i n \theta}
$$

where $r=\left(x_{1}^{2}+x_{2}^{2}\right)^{1 / 2}$ and $\theta=\tan ^{-1}\left(x_{2} / x_{1}\right)$ are polar coordinates, $n$ is an integer, and the $H_{n}$ 's are Hankel functions of the first or second kind of order $n$.

\section{RETERENCES}

Ewing, W. M., W. S. Jardetzky, and F. Press, Elastic Waves in Layered Media, McGraw-Hill Book Company, New York, 1957.

Hayes, M., and R. S. Rivlin, A note on the secular equation for Rayleigh waves, $Z$. Angew. Math. Phys., 18, 80-83, 1962.

Love, A. E. H., The Mathematical Theory of Elasticity, 4th ed., Dover Publications, New York, 1944.

Sternberg, E., On the integration of the equations of motion in the classical theory of elasticity, Arch. Rational Mech. and Analysis, 6, 34-50, 1960.

(Received April 4, 1966.) 